\title{
FIRST, DO NO HARM
}

\section{The risks of overtreating children with epilepsy}

\author{
Eunice Chuang ${ }^{1}$, Marilisa M. Guerreiro ${ }^{4}$, Sara Y. Tsuchie', \\ Angelica Santucci2 ${ }^{2}$ Carlos A. M. Guerreiro ${ }^{4}$, Maria Augusta Montenegro ${ }^{3}$
}

\begin{abstract}
Background: Although overtreatment with antiepileptic drugs contributes to the morbidity associated with epilepsy, many children still are overtreated. Objective: To evaluate if the withdrawal of at least one antiepileptic drug (AED) in children with refractory epilepsy using polytherapy enable a better seizure control. Method: This was a prospective study. Children with refractory epilepsy using at least two AEDs were included. Once the patient, or guardian, agreed to participate in the study, one or more AED were slowly tapered off. The remaining AEDs dosages could be adjusted as needed, but a new AED could not be introduced. Results: Fifteen patients were evaluated, three girls; ages ranging from 3 to 18 (mean $=8.7$ years). After at least one AED withdrawal, two (13.5\%) patients became seizure free, seizures improved $>50 \%$ in $5(33.5 \%)$ patients, did not change in $5(33.5 \%)$, and seizure frequency became worse in $3(20 \%)$. Adverse events improved in 12 patients (80\%). Conclusion: The withdrawal of at least one AED is a valuable option in the treatment of selected children with refractory epilepsy.
\end{abstract}

KEY WORDS: epilepsy, children, antiepileptic drug, overtreatment.

\section{Primeiramente, não causar dano: os riscos do excesso de medicações no tratamento da epilep- sia na infância}

RESUMO - Introdução: Apesar do tratamento excessivo com drogas antiepilépticas (DAE) contribuir para a morbidade associada à epilepsia, muitas crianças ainda são submetidas a politerapia desnecessária. Objetivo: Avaliar se a retirada de pelo menos uma DAE em crianças com epilepsia refratária utilizando politerapia pode proporcionar melhor controle das crises epilépticas. Métodos: Este foi um estudo prospectivo. Crianças com epilepsia refratária em uso de pelo menos duas DAE foram incluídas. Após assinatura do consentimento informado, uma ou mais DAE foram lentamente retiradas. As doses das outras DAE que não foram retiradas poderiam ser ajustadas se necessário, mas uma nova DAE não pode ser introduzida. Resultados: Quinze pacientes foram avaliados, três eram meninas, com idades entre 3 e 18 anos (média=8,7). Após a retirada de pelo menos uma DAE, $2(13,5 \%)$ pacientes ficaram livre de crises, as crises melhoraram em $5(33,5 \%)$, não mudaram em $5(33,5 \%)$ e a frequencia das crises pioraram em $3(20 \%)$ pacientes. Os eventos adversos melhoraram em 12 patientes $(80 \%)$. Conclusão: A retirada de pelo menos uma DAE é uma opção válida no tratamento de crianças com epilepsia refratária.

PALAVRAS-CHAVE: epilepsia, infância, droga antiepiléptica, tratamento excessivo.

Most children with epilepsy become seizure free after the introduction of one antiepileptic drug $(A E D)^{1,2}$. There is universal agreement that the prescription of a single agent at the lowest therapeutic dosage constitutes the best practice in the treatment of epilepsy3,4. This is the only way to avoid the risks of AED overtreatment ${ }^{4}$. Despite that, the use of polytherapy remains, especially if the first AED fails to control the seizures.

It is known that the reduction of one or more AED is possible without an increase in seizure frequency ${ }^{5-7}$. Moreover, AEDs may aggravate pre-existing seizures and trigger new seizure types ${ }^{8}$.

The objective of this study was to evaluate if the withdrawal of at least one AED in children with refractory epilepsy using polytherapy can improve seizure control.

\section{METHOD}

This was a prospective study conducted at the pediatric

Department of Neurology - State University of Campinas SP, Brazil (UNICAMP): ${ }^{1}$ Aluna de Medicina; ${ }^{2}$ Neurologista Infantil; ${ }^{3}$ Professora Doutora; ${ }^{4}$ Professor Titular. Eunice Chuang and Sara Y. Tsuchie are medical students and received a scholarship from CNPq.

Received 4 August 2006. Accepted 30 October 2006.

Marilisa M. Guerreiro, MD, PhD - Department of Neurology - FCM/Unicamp - P.O. Box 6111 - 13083-970 Campinas SP - Brazil. E-mail: guga@fcm.unicamp.br 
epilepsy clinic of our University Hospital from January 2005 to December 2005. Inclusion criteria consisted of age between 1 and 18 years-old, diagnosis of refractory epilepsy, use of at least two AEDs, signature of informed consent approved by the Ethical Committee of our institution.

Once the patient, or guardian, agreed to participate in the study, one or more AEDs were slowly tapered off. The remaining $A E D$ s dosages could be adjusted as needed, but a new AED could not be introduced.

After drug withdrawn we assessed seizure frequency and adverse events. Routine visits were scheduled and patients were instructed to seek medical care at our institution as needed, especially in case of seizure exacerbation.

\section{RESULTS}

Fifteen patients met the inclusion criteria and were included in the protocol, three girls and 10 boys; ages ranging from 3 to 18 (mean=8.7 years). Table 1 shows the characteristics of the patients.

After at least one AED withdrawal, two (13.5\%) patients became seizure free, seizures improved $>50 \%$ in $5(33.5 \%)$ patients, did not change in 5 $(33.5 \%)$, and seizure frequency became worse in 3 (20\%). Adverse events improved in 12 patients (80\%; Table 2).

It should be noted that due to ethical issues, the doses of the remaining AED could be adjusted as needed. This probably contributed to seizure improvement in two patients (patients 2 and 8). However, five patients (patients 9, 10, 12, 13 and 14) presented improvement in seizure control after the withdraw of one AED, without any modification in the remaining AEDs dosages.

\section{DISCUSSION}

There is no question that seizure freedom is the main goal for patients, families and doctors dealing with epilepsy. However, a small percentage of children

Table 1. Characteristics of the patients.

\begin{tabular}{|c|c|c|c|c|c|}
\hline ID & Gender/Age & Etiology of epilepsy & Neuroimaging & Type of epileptic syndrome & Neurological examination \\
\hline 1 & $5 / F$ & Chiari II malformation & Hydrocephalus & Partial & $\begin{array}{l}\text { Developmental delay, } \\
\text { macrocephaly, lower limb } \\
\text { hypotonia and paresis }\end{array}$ \\
\hline 2 & $12 / \mathrm{M}$ & Criptogenic & Normal & Partial & Normal \\
\hline 3 & $3 / F$ & Criptogenic & Normal & Indeterminated & Developmental delay \\
\hline 4 & $15 / \mathrm{M}$ & $\begin{array}{l}\text { Neonatal complications } \\
\text { (mainly hypoglicemia) }\end{array}$ & Gliosis & $\begin{array}{l}\text { Generalized (Lennox- } \\
\text { Gastaut syndrome) }\end{array}$ & $\begin{array}{l}\text { Developmental delay, } \\
\text { tetraparesis }\end{array}$ \\
\hline 5 & $6 / \mathrm{M}$ & Brain malformation & $\begin{array}{l}\text { Bilateral } \\
\text { schizencefaly }\end{array}$ & $\begin{array}{l}\text { Generalized (Lennox- } \\
\text { Gastaut syndrome) }\end{array}$ & $\begin{array}{l}\text { Developmental delay, } \\
\text { tetraparesis, subnormal vision }\end{array}$ \\
\hline 6 & $13 / \mathrm{M}$ & Perinatal complications & $\begin{array}{l}\text { Periventricular } \\
\text { leucomalacia }\end{array}$ & $\begin{array}{l}\text { Generalized (Lennox- } \\
\text { Gastaut syndrome) }\end{array}$ & $\begin{array}{l}\text { Developmental delay, } \\
\text { tetraparesis }\end{array}$ \\
\hline 7 & $3 / \mathrm{M}$ & $\begin{array}{l}\text { Neonatal complication } \\
\text { (mainly prematurity) }\end{array}$ & Hydrocefalus & $\begin{array}{l}\text { Generalized (West } \\
\text { syndrome) }\end{array}$ & $\begin{array}{l}\text { Developmental delay, } \\
\text { tetraparesys }\end{array}$ \\
\hline 8 & $12 / \mathrm{M}$ & Unkown & Normal & Partial & $\begin{array}{l}\text { Developmental delay, } \\
\text { mental retardation }\end{array}$ \\
\hline 9 & $5 / \mathrm{M}$ & $\begin{array}{l}\text { Hypoxic ischemic } \\
\text { Encephalopathy }\end{array}$ & Focal atrophy & $\begin{array}{l}\text { Generalized (Lennox- } \\
\text { Gastaut syndrome) }\end{array}$ & $\begin{array}{l}\text { Developmental delay, } \\
\text { hypotonia }\end{array}$ \\
\hline 10 & $12 / \mathrm{M}$ & Unknown & Normal & $\begin{array}{l}\text { Generalized (Lennox- } \\
\text { Gastaut syndrome) }\end{array}$ & $\begin{array}{l}\text { Developmental delay, } \\
\text { mental retardation }\end{array}$ \\
\hline 11 & $18 / \mathrm{M}$ & Unknown & Normal & $\begin{array}{l}\text { Generalized (Lennox- } \\
\text { Gastaut syndrome) }\end{array}$ & $\begin{array}{l}\text { Developmental delay, } \\
\text { mental retardation }\end{array}$ \\
\hline 12 & $6 / \mathrm{M}$ & Meningoencephalitis & $\begin{array}{l}\text { Multicystic } \\
\text { leucomalacia }\end{array}$ & $\begin{array}{l}\text { Generalized (Lennox- } \\
\text { Gastaut syndrome) }\end{array}$ & $\begin{array}{l}\text { Developmental delay, } \\
\text { tetraparesis and microcephaly }\end{array}$ \\
\hline 13 & $8 / \mathrm{M}$ & $\begin{array}{l}\text { Myoclonic astatic } \\
\text { epilepsy }\end{array}$ & Normal & Generalized & $\begin{array}{l}\text { Developmental delay, } \\
\text { mental retardation }\end{array}$ \\
\hline 14 & $10 / \mathrm{M}$ & Criptogenic & Normal & $\begin{array}{l}\text { Generalized (Lennox- } \\
\text { Gastaut syndrome) }\end{array}$ & $\begin{array}{l}\text { Developmental delay, } \\
\text { mental retardation }\end{array}$ \\
\hline 15 & $4 / F$ & Meningoencephalitis & Normal & $\begin{array}{l}\text { Generalized (Lennox- } \\
\text { Gastaut syndrome) }\end{array}$ & $\begin{array}{l}\text { Developmental delay, mental } \\
\text { retardation, global hypotonia }\end{array}$ \\
\hline
\end{tabular}


Table 2. Adverse events and seizure control after AED withdrawal.

\begin{tabular}{|c|c|c|c|c|c|c|c|}
\hline ID & AED & $\begin{array}{l}\text { Adverse } \\
\text { event }\end{array}$ & $\begin{array}{l}\text { Number of } \\
\text { seizures at } \\
\text { baseline }\end{array}$ & $\begin{array}{l}\text { AED } \\
\text { withdrawn }\end{array}$ & $\begin{array}{l}\text { Adverse event } \\
\text { after AED } \\
\text { withdrawal }\end{array}$ & $\begin{array}{l}\text { Seizures after } \\
\text { AED with- } \\
\text { drawal }\end{array}$ & $\begin{array}{l}\text { Follow- } \\
\text { up }\end{array}$ \\
\hline 1 & $\begin{array}{l}\text { DPH }(6.5 \mathrm{mg} / \mathrm{kg} / \mathrm{d}) \\
\text { PB }(3.6 \mathrm{mg} / \mathrm{kg} / \mathrm{d})\end{array}$ & Apathy & 4 / day & PB & Improved & Worse & - \\
\hline 2 & $\begin{array}{l}\text { CBZ }(15 \mathrm{mg} / \mathrm{kg} / \mathrm{d}) \\
\mathrm{DPH}(5 \mathrm{mg} / \mathrm{kg} / \mathrm{d}) \\
\text { CLB }(5 \mathrm{mg} / \mathrm{d})\end{array}$ & None & 20 / day & $\begin{array}{l}\text { CLB DPH } \\
\uparrow \mathrm{CBZ}(22,5 \mathrm{mg} / \mathrm{kg} / \mathrm{d})\end{array}$ & $\begin{array}{l}\text { No } \\
\text { improvement }\end{array}$ & $\begin{array}{l}\text { Improved } \\
\text { (seizure-free) }\end{array}$ & $\begin{array}{l}12 \\
\text { months }\end{array}$ \\
\hline 3 & $\begin{array}{l}\text { LTG }(6 \mathrm{mg} / \mathrm{kg} / \mathrm{d}) \\
\text { VA }(25 \mathrm{mg} / \mathrm{kg} / \mathrm{d}) \\
\text { CLB }(20 \mathrm{mg} / \mathrm{d})\end{array}$ & None & $1 /$ week & $\begin{array}{l}\text { VA } \\
\text { CLB } \\
\uparrow \text { LTG (200 mg) }\end{array}$ & Improved & $\begin{array}{l}\text { No } \\
\text { improvement }\end{array}$ & $\begin{array}{l}10 \\
\text { months }\end{array}$ \\
\hline 4 & $\begin{array}{l}\text { VA }(29 \mathrm{mg} / \mathrm{kg} / \mathrm{d}) \\
\text { LTG }(8,8 \mathrm{~kg} / \mathrm{d}) \\
\text { TPM }(5,8 \mathrm{mg} / \mathrm{kg} / \mathrm{d}) \\
\text { CZP }(0,5 \mathrm{mg} / \mathrm{kg} / \mathrm{d}) \\
\text { NTZ }(5 \mathrm{mg} / \mathrm{kg} / \mathrm{d})\end{array}$ & Somnolence & 2-3/day & $\begin{array}{l}\text { CZP } \\
\text { NTZ }\end{array}$ & Improved & $\begin{array}{l}\text { No } \\
\text { improvement }\end{array}$ & $\begin{array}{l}6 \\
\text { months }\end{array}$ \\
\hline 5 & $\begin{array}{l}\text { CLB }(10 \mathrm{ml} / \mathrm{d}) \\
\operatorname{LTG}(200 \mathrm{mg} / \mathrm{d}) \\
\operatorname{VGB}(1000 \mathrm{mg} / \mathrm{d})\end{array}$ & None & $20 /$ day & VGB & $\begin{array}{l}\text { No } \\
\text { improvement }\end{array}$ & Worse & - \\
\hline 6 & $\begin{array}{l}\text { NTZ }(15 \mathrm{mg} / \mathrm{d}) \\
\text { CBZ }(1000 \mathrm{mg} / \mathrm{d}) \\
\text { PB }(100 \mathrm{mg} / \mathrm{d})\end{array}$ & $\begin{array}{l}\text { Somnolence, } \\
\text { apathy, } \\
\text { poor school } \\
\text { attendance/ } \\
\text { performance }\end{array}$ & 3-10 / day & PB & Improved & $\begin{array}{l}\text { No } \\
\text { improvement }\end{array}$ & $\begin{array}{l}12 \\
\text { months }\end{array}$ \\
\hline 7 & $\begin{array}{l}\text { VA (40 mg/kg/d) } \\
\text { CLB ( } 2 \mathrm{mg} / \mathrm{kg} / \mathrm{day}) \\
\text { VGB (100 mg/kg/day) }\end{array}$ & $\begin{array}{l}\text { Somnolence, } \\
\text { apathy }\end{array}$ & $\begin{array}{l}\text { Daily } \\
\text { seizures }\end{array}$ & $\begin{array}{l}\text { VGB } \\
\uparrow \text { VA }(50 \mathrm{mg} / \mathrm{kg} / \mathrm{day}) \\
\uparrow \mathrm{CLB}(2.2 \mathrm{mg} / \mathrm{kg} / \mathrm{d})\end{array}$ & Improved & $\begin{array}{l}\text { No } \\
\text { improvement }\end{array}$ & $\begin{array}{l}8 \\
\text { months }\end{array}$ \\
\hline 8 & $\begin{array}{l}\text { VA ( } 18 \mathrm{mg} / \mathrm{kg} / \text { day }) \\
\text { FB ( } 5 \mathrm{mg} / \mathrm{kg} / \text { day }) \\
\text { CZP (0.5 mg/day) }\end{array}$ & $\begin{array}{l}\text { Somnolence, } \\
\text { apathy }\end{array}$ & 15 / day & $\begin{array}{l}\text { PB } \\
\text { CZP } \\
\uparrow \text { VA }(25 \mathrm{mg} / \mathrm{kg} / \text { day })\end{array}$ & Improved & $\begin{array}{l}\text { Improved } \\
\text { (seizure-free) }\end{array}$ & $\begin{array}{l}12 \\
\text { months }\end{array}$ \\
\hline 9 & $\begin{array}{l}\text { FB ( } 3 \text { mg/kg/day) } \\
\text { VA ( } 56 \text { mg/kg/day) } \\
\text { CLB ( } 40 \text { mg/day) }\end{array}$ & $\begin{array}{l}\text { Somnolence, } \\
\text { apathy }\end{array}$ & $5 /$ day & PB & Improved & $\begin{array}{l}\text { Improved: } 0-2 \\
\text { seizures / day }\end{array}$ & $\begin{array}{l}6 \\
\text { months }\end{array}$ \\
\hline 10 & $\begin{array}{l}\text { LTG (350 mg/day) } \\
\text { CLB (45 mg/day) } \\
\text { TPM (150 mg/day) }\end{array}$ & $\begin{array}{l}\text { Somnolence, } \\
\text { apathy }\end{array}$ & 4 / day & TPM & Improved & $\begin{array}{l}\text { Improved } \\
80 \%\end{array}$ & $\begin{array}{l}12 \\
\text { months }\end{array}$ \\
\hline 11 & $\begin{array}{l}\text { CZP (3.5 mg/day) } \\
\text { CBZ (900 mg/day) } \\
\text { VA ( } 250 \text { mg/day) }\end{array}$ & None & 9 / week & VA & $\begin{array}{l}\text { No } \\
\text { improvement }\end{array}$ & Worse & - \\
\hline 12 & $\begin{array}{l}\text { DPH (7 mg/kg/day) } \\
\text { PB (3.6 mg/kg/day) }\end{array}$ & $\begin{array}{l}\text { Somnolence, } \\
\text { apathy, gun } \\
\text { hypertrophy, } \\
\text { hypertricosis }\end{array}$ & 30 / day & DPH & Improved & $\begin{array}{l}\text { Improved: } 0-3 \\
\text { seizures/day }\end{array}$ & 2 month \\
\hline 13 & $\begin{array}{l}\text { LTG (400 mg/day) } \\
\text { CZP (3 mg/day) }\end{array}$ & $\begin{array}{l}\text { Somnolence, } \\
\text { apathy }\end{array}$ & 2 / week & LTG & Improved & $\begin{array}{l}\text { Improved: } \\
1 / \text { month }\end{array}$ & 3 month \\
\hline 14 & $\begin{array}{l}\text { TPM (3 mg/kg/day) } \\
\text { CLB ( } 20 \mathrm{mg} / \text { day) } \\
\text { VA ( } 39 \mathrm{mg} / \mathrm{kg} / \text { day) }\end{array}$ & $\begin{array}{l}\text { Somnolence, } \\
\text { apathy, lost of } \\
\text { weight }\end{array}$ & 15 / day & TPM & Improved & $\begin{array}{l}\text { Improved: } 5 \\
\text { seizures / day }\end{array}$ & 1 month \\
\hline 15 & $\begin{array}{l}\text { PB ( } 5 \text { mg/kg/dia) } \\
\text { VA ( } 30 \mathrm{mg} / \mathrm{dkg} / \text { day) } \\
\text { CZP ( } 1 \mathrm{mg} / \text { day) }\end{array}$ & $\begin{array}{l}\text { Somnolence, } \\
\text { apathy }\end{array}$ & 4 / day & PB & Improved & $\begin{array}{l}\text { No } \\
\text { improvement }\end{array}$ & $\begin{array}{l}3 \\
\text { months }\end{array}$ \\
\hline
\end{tabular}

AED, antiepileptic drug; CBZ, carbamazepine; CLB, clobazam; CZP, clonazepam; DPH, phenytoin; PB, phenobarbital; TPM, topiramate; VA, valproate; LTG, lamotrigina; NTZ, nitrazepam; VGB, vigabatrin. 
will present refractory seizures, and will not become seizure-free despite adequate AED treatment ${ }^{9-11}$. Epilepsy generally demands prolonged AED treatment which is often associated with drug toxicity, especially when there is the use of an excessive - and sometimes unnecessary - number of AEDs ${ }^{4,12-14}$.

Although there is increasing awareness that overtreatment with AEDs contributes to the morbidity associated with epilepsy ${ }^{4}$, many children still are overtreated. One possible explanation can be the fact that seizures are probably one of the most frightening event a parent can experience. For that reason, parents often will take the child with refractory epilepsy to as many doctors as needed to have their seizures controlled. In addition, most parents will try almost anything if there is a small hope of seizure freedom. As for doctors, it is sometimes too hard to resist the family's desperate question "Can we add a new drug?"

Our findings show that the reduction of one or more AED is possible without an increase in seizure frequency, which is in keeping with other studies ${ }^{5-7}$. In addition, two patients became seizure free.

It should be kept in mind that the outcome of epilepsy treatment should not be measured only by the percentage of seizure reduction ${ }^{15}$. Quality of life is related not only to seizure control, but also to adverse events. We found that the withdraw of one AED provided a considerable improvement in the adverse events of five $(33.5 \%)$ patients despite of no improvement in seizure control. This enable a better quality of life, and families referred that their children were feeling much better, despite no seizure control.

Epilepsy is a frightening condition, and some families cannot cope with a single febrile seizure. However, it is surprising how well many parents of a severely handicapped child - and above all, the children themselves - can sometimes cope very well with seizures. After the protocol, the substantial improvement in drug related adverse events enabled one of our patients with cerebral palsy to go back to school (patient 6).
One possible limitation of our study was that, according to ethical issues, the remaining AEDs dosages could be adjusted as needed. Higher doses of the remaining AED probably contributed to seizure improvement in two patients. However, five patients presented improvement in seizure control after the withdraw of one AED, without any modification in the remaining AEDs dosages.

We conclude that although a larger sample is needed in order to confirm our findings, the reduction of one AED in selected children with refractory epilepsy can be associated with less adverse events and better quality of life without worsening of seizure frequency. In addition, a few patients may present an improvement in seizure control.

\section{REFERENCES}

1. Camfield CS, Camfield P, Gordon K, Smith B, Dooley J. Outcome of childhood epilepsy: a population-based study with a simple scoring system for those treated with medication. J Pediatr 1993;122:861-868.

2. Silva M, MacArdle B, MaGowan M, et al. Randomised comparative monotherapy trial of phenobarbitone, phenytoin, carbamazepine, or sodium valproate for newly diagnosed childhood epilepsy. Lancet 1996; 347:709-713

3. Baulac M. Rational conversion from antiepileptic polytherapy to monotherapy. Epileptic Disord 2003;5:125-132

4. Holmes GL. Overtreatment in children with epilepsy. Epilepsy Res 2002;52:35-42.

5. Fischbacher E. Effect of reduction of anticonvulsants on wellbeing. $\mathrm{Br}$ Med J (Clin Res Ed) 1982;285:423-424.

6. Schmidt D. Reduction of two-drug therapy in intractable epilepsy. Epilepsia 1983;24:368-376.

7. Alvarez N. Discontinuance of antiepileptic medications in patients with developmental disability and diagnosis of epilepsy. Am J Ment Retard. 1989;93:593-595.

8. Guerrini R, Belmonte A, Genton P. Antiepileptic drug-induced worsening of seizures in children. Epilepsia. 1998;39(Suppl 3):S2-S10.

9. Ko TS, Holmes GL. EEG and clinical predictors of medically intractable childhood epilepsy. Clin Neurophysiol 1999;110:1245-1251.

10. Huttenlocker PR, Hapke RJ. A follow-up study of intractable seizures in childhood. Ann Neurol 1990;28:699-705.

11. Ferngren H, Akerstrom I, Rane A. Mono or polypharmacotherapy in institutionalized epileptic children with severe mental retardation? A team approach for optimizing antiepileptic therapy. Acta Paediatr Scand 1991;80:458-465

12. Dooley J, Gordon K, Camfield C, Smith E. Discontinuation of anticonvulsant therapy in children free of seizures for 1 year: a prospective study. Neurology 1996;46:969-974.

13. Shinnar S, Berg AT, Moshé SL, et al. Discontinuing antiepileptic drugs in children with epilepsy:a prospective study. Ann Neurol 1994;35 534-545.

14. Shorvon SD, Reynolds EH. Unnecessary polypharmacy for epilepsy. Br Med J 1977;1:1635-1637.

15. Vickery BG, Hay R, Engel J. Outcome assessment for epilepsy surgery: the impact of measuring health-related quality of life. Ann Neurol 1995; $37: 158-166$ 